\title{
Rehabilitación psicosocial para enfermos mentales graves en contextos rurales: alternativas a los modelos tradicionales
}

RESUMEN: El artículo analiza los servicios comunitarios de atención psicosocial para pacientes con enfermedad mental crónica en los contextos rurales. El propósito es determinar si los enfoques de atención tradicionales se adaptan al ámbito rural, así como analizar las alternativas existentes a los modelos tradicionales. Se proponen unos criterios que aseguren la eficacia de las intervenciones en las áreas rurales.

Palabras clave: Rehabilitación psicosocial, rural, enfermo mental crónico, eficacia.

\section{Introducción}

Existe un porcentaje significativo de enfermos mentales graves que viven en áreas rurales en España. Aunque en numerosos artículos y publicaciones se han descrito y definido el tipo de servicios de salud mental más adecuados para esta población $(1,2,3)$, existen muy pocas referencias acerca de cómo adaptarlos a los ámbitos rurales.

\begin{abstract}
The objetive is to describe the psychosocial community services for seriously mentally ill persons living in rural settings. This paper describes the adaptation of traditional models to determine its efficacy in rural areas. It is described alternative programs developed for patients in rural communities. It is proposed a group of criteria for the psychosocial services in rural areas.
\end{abstract}

Key words: Psychosocial rehabilitation, rural, Chronic mental patients, effectiveness.

Ya ha quedado sobradamente demostrado que los programas más adecuados para los enfermos mentales graves son los equipos de salud mental comunitarios, entre los que se encuentran los programas de rehabilitación psicosocial y apoyo comunitario (4-8). Estos programas se basan en el desarrollo e implementación de las habilidades que permitan al paciente vivir en 
INFORMES

la comunidad de la forma más integrada posible $(9,10)$. Las intervenciones psicosociales son una herramienta imprescindible para los casos más graves, que permiten alcanzar un mayor nivel de autonomía y conseguir una mayor calidad de vida (11).

Sin embargo, y aunque hay evidencias de la utilidad de los equipos de atención comunitaria a enfermos mentales graves en contextos rurales (12-14), hay una escasez de publicaciones sobre la manera de implementarlos considerando las características diferenciales que conlleva el contexto rural.

El objetivo del presente artículo es describir cuáles son los modelos de atención psicosocial más adecuados para el contexto comunitario rural. El ámbito rural presenta particularidades que obligan a plantearse la adaptación de los modelos de atención tradicionales ya que, previsiblemente, no se obtendrán los mismos resultados que en las zonas urbanas. Así, por ejemplo, los servicios de salud mental de las áreas rurales están a menudo fragmentados y poco coordinados, permanecen inaccesibles para una parte importante de la población y existe mayor escasez de profesionales trabajando en los equipos. Como consecuencia, muchos pacientes graves quedarán fuera del sistema de atención y no se beneficiarán de los programas de rehabilitación psicosocial, aumentando su deterioro y sus discapacidades.

De hecho, y a pesar de que han pasado veinte años de trabajo en todo el mundo sobre diferentes aspectos acerca de los servicios de salud mental comunitaria, no existe una taxonomía de servicios y prestaciones de salud mental que permita comparar los componentes, servicios y estándares en distintos ámbitos urbanos y rurales.

Recientes trabajos están empezando a remediar este déficit, tratando de definir las prestaciones básicas que deben contemplarse para asegurar unos estándares de calidad adecuados en los servicios $(15,16)$. En estos trabajos se reconoce la necesidad de contar con sistemas estandarizados de descripción y clasificación de servicios de salud mental, que favorezca la comparación entre diferentes áreas de cobertura. Sin embargo estos documentos técnicos no van dirigidos a contextos rurales y por tanto no cuentan con las características diferenciales de dicho contexto, dificultando el análisis específico de esta situación. Sin duda, el modelo de asistencia (derivaciones, coordinaciones, seguimiento, atención en crisis, continuidad, accesibilidad y disponibilidad de cuidados) en el área rural va a venir definido por las condiciones asociadas a dicho contexto más que por el tipo de prestaciones terapéuticas o rehabilitadoras que es necesario asignar a los pacientes.

Este artículo revisa los modelos de atención psicosocial que se han implementado en distintos contextos rurales y describe los ingredientes que son necesarios considerar en este ámbito para asegurar una mayor calidad de los servicios. Se hace una mención especial a los programas de tratamiento asertivo comunitario o de seguimiento intensivo en la comunidad, considerando que parte de sus principios y metodología se adaptan a la 
realidad rural en gran medida (actuaciones in vivo, desplazamiento de profesionales, prevención de abandonos y contactos frecuentes con los pacientes).

\section{Definición de contexto rural}

Con el objetivo de analizar qué elementos del entorno influyen y de qué manera en la atención al enfermo mental en el ámbito rural, un primer paso es tratar de definir lo que se entiende por «contexto rural» y comprender la naturaleza de la diferencia entre los modelos de salud mental urbanos y rurales.

McDonel y otros (17) hicieron una revisión del tema y analizaron las ventajas y las desventajas del contexto rural en la planificación de servicios de salud mental. Como desventajas señalan: mayores barreras para el transporte, más escasez de personal cualificado, mayor estigma hacia la enfermedad mental, menor variedad y mayor fragmentación de servicios. También encontraron una menor cantidad de recursos comunitarios y mayores índices de pobreza y desempleo en comparación con los contextos urbanos. El aislamiento de los profesionales, el menor acceso a la supervisión clínica y la ausencia de formación continuada también aparece como un denominador común en este ámbito, lo cual puede contribuir a una menor aceptación y difusión de nuevas tecnologías de tratamiento. La escasez de recursos también contribuye a una menor colaboración entre los profesionales de salud mental, debido a la presión asistencial, lo que convierte a los clínicos del contexto rural en «clínicos generalistas», perdiéndose la es- pecialización imprescindible que conlleva la atención psicosocial al enfermo mental crónico. En lo que se refiere a los pacientes, no acuden a los servicios por el miedo realista a que se pierda la confidencialidad. Este temor a acudir a los servicios de salud mental sumado a la dificultad de los más graves para articular demandas, facilita que en el contexto rural los pacientes más graves resulten «invisibles». Por otro lado, se ha comprobado que la evaluación de programas es menos común en el ámbito rural que en el urbano, quizás no solo por la escasez de los mismos o la formación de los profesionales, si no por la percepción de estar dando una calidad asistencial inferior a la esperada.

Como ventajas del ámbito rural el grupo de McDonel (17) incluyen: mayor accesibilidad a las redes informales sociales y familiares y mayor conocimiento de los recursos que ofrece la comunidad. Concluyen su revisión señalando que en el área rural es más probable desarrollar redes sociales más tupidas, en las que los profesionales y las personas de la red pueden complementarse más fácilmente. Esto puede ser una ventaja cuando es necesario dar una respuesta rápida para prevenir una crisis, pero puede ser una desventaja cuando se pierde la privacidad y la distancia profesional.

Sullivan y colaboradores (18) examinaron las diferencias demográficas, clínicas y en el uso de servicios entre pacientes que vivían en áreas rurales y urbanas. Entre las principales dificultades que encontraron en el ambiente rural estaban un menor rango de servicios de atención, mayores dificultades para reclutar y retener a los 
profesionales, mayores distancias de desplazamiento y ausencia de transporte público, dificultad para contactar con los pacientes debido a que un porcentaje no desdeñable no tiene teléfono. En cuanto a las diferencias encontradas entre los pacientes rurales y urbanos encontraron que: los pacientes habían completado en menor medida una educación reglada, vivían con la familia un $89 \%$ de los casos comparados con un $69 \%$ de los urbanos, los pacientes rurales habían recibido menos cuidados comunitarios de salud mental (atención individualizada y visitas a domicilio - un $40 \%$ comparado con un $65 \%$ de los urbanos -), los pacientes rurales usaban en mayor medida servicios hospitalarios de emergencia y acudían más al médico de cabecera que los pacientes urbanos.

Santos y otros (13) han señalado el fenómeno de puerta giratoria como definitorio del contexto rural. Estos autores encuentran que los individuos de áreas rurales entran frecuentemente en el sistema de atención mediante hospitalizaciones. Luego tras el alta, vuelven a su comunidad rural y pierden el seguimiento desde salud mental, permaneciendo sin tratamiento durante un tiempo hasta que vuelven a requerir atención de emergencia hospitalaria.

En nuestro país, Simón (19) ha analizado el contexto rural en relación a la accesibilidad de los servicios de salud mental. Según Simón dos son las características esenciales de lo que se entiende por «rural»: 1) la lejanía y la dispersión geográfica que determina la dificultad de acceso a las prestaciones; 2) el contexto sociocultural. Continúa señalando que los retos planteados por estas características están en la planificación de dispositivos y programas que garanticen la accesibilidad en la incorporación del contexto sociocultural en el diseño y ejecución de las actividades de rehabilitación psicosocial.

Se han encontrado niveles de recaída más elevados en las zonas rurales (20) y ratios de utilización de servicios de salud mental más bajos (21). El dato de que los enfermos mentales de las zonas rurales utilizan menos servicios de salud mental se repite en la mayoría de los estudios (17, $18,22,21)$ y puede responder a un problema de accesibilidad inherente a lo rural, más que a condiciones culturales, demográficas, clínicas o asistenciales (23, 24).

Estos factores son claves a la hora de implantar programas de salud mental sin pararse a pensar que lo que funciona en el ámbito urbano puede que no funcione en el ámbito rural.

A pesar de todo lo dicho, algunos autores en la línea comentada por McDonel (17) siguen señalando aspectos que se consideran positivos para la asistencia al paciente en el ámbito rural. Así, Simón (19) considera como positivos: el sentido de comunidad que genera un gran potencial de ayuda para el proceso de inserción, el mantenimiento de la red familiar extensa, una mayor tolerancia a las personas que padecen enfermedades mentales, mayor abanico de roles sociales valiosos y oportunidades de practicarlos y una mayor localización del paciente que facilita el seguimiento y la continuidad de cuidados.

Desde nuestra experiencia, sin embargo, creemos que estas ventajas enumeradas por algunos autores se traducen en des- 
ventajas en la práctica asistencial diaria. Así, por ejemplo, el mantenimiento y aceptación del paciente en el medio familiar genera una gran tolerancia de comportamientos inadecuados del enfermo provocando una gran resistencia a cualquier cambio planteado desde fuera. Esto implica que la familia relativiza una serie de conductas dentro de la casa que mantienen la situación de deterioro y que impiden no sólo el reconocimiento de la problemática, sino las intervenciones que tratan de modificar aquellas condiciones de vida que mantienen la cronicidad. Estas actitudes son manifestadas u ocultadas a los demás vecinos, generando expectativas poco claras sobre el enfermo mental, que llevan a aceptar o a rechazar tales comportamientos y a caer en actitudes de tolerancia o crítica poco favorables para su integración real. En lo que se refiere a la aceptación comunitaria y a la posibilidad de ejercer mayor número de roles, podemos comprobar que, aunque en el ámbito rural casi nadie manifiesta públicamente un rechazo social al enfermo mental, existe un actitud de rechazo sutil, manifestada en comportamientos cotidianos (no ven la necesidad de participar en el proceso de rehabilitación, recelos ante la participación activa del paciente en recursos sociales, expectativas de incapacidad del paciente para desarrollar un empleo, apartarle de las decisiones importantes, etc.).

\section{Modelos de atención psicosocial para en-} fermos mentales graves en contextos rurales

Los modelos comunitarios de salud mental son claramente superiores a los anteriores modelos hospitalarios. Hay evidencias en todo el mundo de que los servicios comunitarios reducen las hospitalizaciones, incrementan la satisfacción de los pacientes y no suponen un incremento del

Tabla1. Características del contexto rural en salud mental

\begin{tabular}{|c|c|c|c|c|}
\hline PROFESIONALES & $\begin{array}{l}\text { SERVICIOS DE } \\
\text { ATENCIÓN }\end{array}$ & $\begin{array}{l}\text { ÁMBITO } \\
\text { SOCIOCULTURAL }\end{array}$ & PACIENTES & VENTAJAS \\
\hline $\begin{array}{l}\text { * Escasez de perso- } \\
\text { nal cualificado } \\
\text { * Aislamiento profe- } \\
\text { sional } \\
\text { * Escasa supervisión } \\
\text { clínica } \\
\text { * Ausenciadeforma- } \\
\text { ción continuada } \\
\text { * Poca colaboración } \\
\text { entreprofesionales } \\
\text { * S o b r e c a r g a } \\
\text { asistencial } \\
\text { * Dificultad para re- } \\
\text { clutar y mantener a } \\
\text { los profesionales }\end{array}$ & $\begin{array}{l}\text { * Poca variedad } \\
\text { * Poca cantidad } \\
\text { * Fragmentación } \\
\text { * No evaluación de } \\
\text { eficacia o calidad } \\
\text { asistencial } \\
\text { * Inaccesibilidad } \\
\text { * Atención primaria } \\
\text { atiende a un gran } \\
\text { porcentaje de en- } \\
\text { fermos mentales }\end{array}$ & $\begin{array}{l}\text { * Estigma hacia la } \\
\text { enfermedad mental } \\
\text { * Indices de pobreza } \\
\text { y desempleo } \\
\text { * Ausencia de trans- } \\
\text { porte } \\
\text { * Mayores distancias } \\
\text { en desplazamien- } \\
\text { tos } \\
\text { * Dispersión geográ- } \\
\text { fica } \\
\text { * Tolerancia hacia } \\
\text { conductas proble- } \\
\text { ma } \\
\text { * Menor cantidad de } \\
\text { recursos comuni- } \\
\text { tarios }\end{array}$ & $\begin{array}{l}\text { * Acuden a salud } \\
\text { mental en menor } \\
\text { medida } \\
\text { * Miedo a perder la } \\
\text { confidencialidad } \\
\text { * Dificultad paracon- } \\
\text { tactar con el pa- } \\
\text { ciente } \\
\text { * Mayores niveles de } \\
\text { recaída } \\
\text { * Viven con la fami- } \\
\text { lia } \\
\text { * Incapacidad para } \\
\text { articular sus de- } \\
\text { mandas }\end{array}$ & $\begin{array}{l}\text { * Mayor accesibili- } \\
\text { dad a redes socia- } \\
\text { les y familiares } \\
\text { * Mayor aceptación } \\
\text { del enfermo mental } \\
\text { * Agilidad en locali- } \\
\text { zar al paciente }\end{array}$ \\
\hline
\end{tabular}


INFORMES

coste económico (25). Sin embargo, los datos actuales no permiten generalizar estos resultados a las áreas rurales. Teniendo en cuenta las características antes comentadas no parece descabellado argumentar la necesidad de seguir asegurando la eficacia del modelo comunitario en relación al ámbito rural.

Es difícil hacer una descripción completa y fiable del desarrollo de la rehabilitación psicosocial en los ámbitos rurales. Hay escasos informes sobre el tema y tampoco abunda la información sobre la metodología y los resultados de los programas de rehabilitación para los enfermos mentales graves de estos ámbitos.

$\mathrm{Al}$ intentar recoger información sobre este tema surgen las siguientes dificultades: a) aparecen programas con distinta denominación que hacen funciones similares; b) el desarrollo de programas específicos de rehabilitación psicosocial dentro del recurso correspondiente es escaso, limitándose a dar un «apoyo comunitario general»; c) no hay delimitación clara de objetivos, actividades asistenciales o personal necesario en el equipo; d) en algunos programas no se realiza rehabilitación psicosocial, limitándose a cubrir aspectos de tratamiento a pesar de considerarse dicho programa como de intervención comunitaria. Posiblemente esto es consecuencia de la necesidad de adaptación al medio local.

A continuación se describen algunos de los modelos de atención descritos en la literatura y llevados a cabo en contextos rurales. Sólo se han tenido en cuenta aquellos modelos que contienen en su metodología los ingredientes básicos de la aten- ción comunitaria y que contemplan la atención psicosocial entre sus acciones u objetivos. Por tanto, sólo se describen aquellos que cumplan con los siguientes principios (26): a) integralidad; b) continuidad; c) trabajo en el entorno; d) perspectiva rehabilitadora.

Para revisar cada modelo se seguirá la siguiente estructura: fundamentación, objetivos, método, perfil de pacientes, personal, actividades, resultados y consideraciones.

Workman y otros (27) desarrollan un programa denominado «satélite» que provee servicios de salud mental a pacientes que viven en áreas rurales. Da cobertura a una población de 150.000 habitantes y a 8 municipios. Las poblaciones están alejadas entre $91 \mathrm{Km}$ y $160 \mathrm{Km}$ del principal centro de atención psiquiátrica. El equipo hace una media de desplazamientos de $2.500 \mathrm{Km}$. al mes y viaja a cada sitio como mínimo una vez al mes. Las comunidades locales facilitan espacio físico, voluntariado, teléfono, recepcionista y refrescos. Los pacientes reciben tratamiento en su comunidad tratando de incrementar la adherencia al tratamiento, el compromiso y colaboración de la familia con el equipo y el mantenimiento del paciente en la comunidad.

El perfil de pacientes engloba trastornos psicóticos, especialmente esquizofrénicos con cuadros de discapacidades graves asociadas. El equipo incluye 1 psiquiatra, 2 «técnicos de salud mental» y 1 enfermero/a. Provee una gama de servicios que consisten en: farmacoterapia, psicoterapia individual y familiar, terapia de grupo, case-mangment, derivación a servicios 
especializados (médicos, psicológicos y sociales), psicoeducación, intervención en crisis y medicación depot.

Se plantea este modelo de equipo móvil para aquellos pacientes que no pueden acudir a los centros psiquiátricos ya que viven en localidades poco accesibles (grandes distancias y ausencia de transporte). Sin embargo, este modelo presenta algunas carencias. Habría que aclarar qué tipo de pacientes son y no son adecuados para este tipo de atención. Por otro lado, habría que investigar el intervalo mínimo para hacer un seguimiento adecuado de pacientes con distinto diagnóstico. También es necesario determinar el tipo de intervenciones que son más eficaces no solo para prevenir hospitalizaciones sino también para favorecer el funcionamiento social del paciente. Estos aspectos contribuirían a proveer un servicio más integral y psicosocial en este equipo «satélite».

Santos y su grupo (13) desarrollaron una adaptación del tratamiento asertivo comunitario (PACT) al ámbito rural con el objetivo de evaluar el efecto del programa en las hospitalizaciones y en el coste del servicio. Las comunidades rurales incluidas en el programa cumplían 2 características: estar en las afueras de un área urbana de cobertura y tener un población de menos de 2.500 habitantes.

El PACT consiste en un abordaje comunitario que se lleva a cabo de forma intensiva en el medio de los pacientes mediante actuaciones «in vivo». El equipo normalmente mantiene el contacto con el paciente y pasa más del $75 \%$ de su tiempo en el lugar de residencia del mismo, con el objetivo de guiarle en la prevención de sus crisis y su inserción en la comunidad. Exis- ten numerosos estudios que muestran la eficacia de los PACT en mejorar las condiciones de vida de los pacientes (28-30).

Los pacientes atendidos en el programa de Santos incluían los siguientes criterios: diagnóstico de trastorno mental severo, historia de numerosos ingresos psiquiátricos y asistencia de larga duración en los servicios de salud mental.

El equipo consistía en un psiquiatra y dos enfermeras especializadas en atención domiciliaria y trabajo social. El psiquiatra era el responsable del tratamiento y del manejo clínico, las enfermeras se encargaban del resto del trabajo multidisciplinar (trabajo social, rehabilitación y atención en el domicilio). Los pacientes eran visitados en su casa por el psiquiatra. En las áreas en las que los pacientes vivían unos cerca de otros el equipo les proporcionaba transporte hasta un lugar común, como un consultorio médico o una iglesia.

Las actividades desarrolladas consistían en: administración de medicación, facilitar el acceso a recursos y psicoeducación. El abordaje era individualizado y continuado en el tiempo.

La intervención arrojó los siguientes resultados: reducción en los días de hospitalización del $79 \%$, reducción del $69 \%$ en el número de hospitalizaciones por año y reducción del $52 \%$ en el coste estimado por paciente (calculado según el coste anual de cada paciente incluido en el programa).

Este programa es un buen ejemplo de adaptación al ámbito rural. Se puede considerar como un modelo ideal de trabajo en la comunidad y con la comunidad, ya que la mayoría de las intervenciones desa- 
INFORMES

rrolladas consistían en trabajar con los apoyos comunitarios de los pacientes (familia, amigos, vecinos, etc.) para conseguir su mantenimiento en la comunidad evitando las crisis. Como problemas específicos asociados al ámbito rural señalaron la dificultad de proveer un servicio de emergencia de 24 horas y el llevar un registro escrito de los pacientes, debido al tiempo que el equipo pasa viajando.

Como déficits de esta experiencia se pueden señalar que no recogen medidas importantes como por ejemplo calidad de vida, desempeño psicosocial o utilización de recursos, aspectos clave para asegurar la eficacia de un abordaje integral en el ámbito rural.

Husted (12) ha evaluado la eficacia de un programa para personas con enfermedad mental crónica en áreas rurales. Se proporciona atención a cinco áreas rurales mediante contactos individualizados en las casas o en la comunidad de los pacientes. Los pacientes atendidos tenían diagnóstico de Esquizofrenia, T.Bipolar, Depresión recurrente, T.de Personalidad y un mínimo de dos hospitalizaciones durante los dos últimos años.

Las intervenciones se llevaban a cabo de forma individualizada en las casas y mediante grupos de apoyo mensuales. Se provee tratamiento farmacológico, atención en crisis y atención social.

El equipo consiste en nueve paraprofesionales pertenecientes a la comunidad en la que trabajan. Tienen titulación en varias áreas: psicología, educación, trabajo social y enfermería.

Como resultados encontraron un reducción en el número de hospitalizacio- nes y en el número de días hospitalizados. A destacar de este modelo está el hecho de que los trabajadores forman parte de la comunidad, lo que puede ayudar a integrar el equipo y los pacientes en la comunidad.

El equipo de McDonel (17) hizo una evaluación de la adaptación del modelo de atención asertivo comunitario al medio rural y describió las barreras que surgen al adaptar los modelos a este ámbito. Diseñaron dos equipos comunitarios. Uno consistía en dos tutores o «case-managers» (CM) con formación en salud mental y un supervisor en el centro de salud mental. El otro equipo consistía en cuatro CM sin formación específica en salud mental y un supervisor con funciones administrativas. Los pacientes atendidos presentaban diagnóstico de trastorno esquizofrénico y trastorno de personalidad, más de un ingreso psiquiátrico y deterioro en su funcionamiento social durante los dos últimos años (trabajo, red social, autonomía básica y economía). Los equipos fueron entrenados en el modelo asertivo comunitario. Las prestaciones que se daban correspondían con la filosofía del PACT: tratamiento y rehabilitación, atención a domicilio, bajo ratio profesional-pacientes y continuidad de cuidados.

Una conclusión a la que llegaron es que en el contexto rural se hacen menos visitas a domicilio que en el urbano, lo que puede mermar la eficacia de los programas. Lo determinante parece ser la frecuencia de visitas, mas que la duración de las mismas. Entre los problemas para adaptar el programa al ámbito rural se encontraron los siguientes: los cm no estaban formados en los principios básicos del 
modelo, el número de visitas a domicilio fue menor del esperado debido al gasto generado por el transporte, no se hacían reuniones regulares con el supervisor para organizar el trabajo, los CM no estaban integrados en la unidad de salud mental de referencia por lo que no podían desarrollar acciones conjuntas con el equipo (clínicas, sociales, ocupacionales, etc.), los CM estaban aislados del resto del equipo siendo inexistentes las interacciones o consultas con el psiquiatra o psicólogo.

Los autores concluyen que el modelo asertivo comunitario podría ser un modelo adecuado para el contexto rural, pero solo si se aplica correctamente. El principal elemento que contribuiría al fracaso del modelo sería la implantación parcial del mismo. En la línea desarrollada por Teague (31) es necesario desarrollar un protocolo para evaluar la fidelidad de los principios asociados a los programas en el ámbito rural. De lo contrario modelos validados en otros ámbitos no tienen por qué arrojar los mismos resultados en el ámbito rural e incluso podrían incrementar el coste general de los servicios.

\section{Adaptación a la realidad rural: Venta- jas y limitaciones}

Como vemos hay una variedad en el tipo y la forma en que los equipos de atención psicosocial al enfermo mental crónico han sido implementados en el ámbito rural. De entre todas las experiencias habidas vamos a considerar algunos modelos que, en la práctica, pueden adecuarse a lo que hemos definido como «lo rural». Aunque conviene señalar que existen di- ferencias entre cada modelo, comparten el aspecto de que se trata de modelos comunitarios apropiados para los servicios públicos.

(1) Equipos comunitarios multidisciplinares o equipos genéricos.

(2) Equipos genéricos con un equipo de PACT complementario.

(3) Equipos genéricos con funciones de PACT añadidas.

(4) Equipos genéricos con un equipo de atención a domicilio.

(5) Equipos genéricos con especialistas integrados en el equipo.

Vamos a considerar cada uno de estos modelos según la evidencia existente y sus relativas ventajas y desventajas (32).

\section{(1) Equipos comunitarios multidisciplina- res o equipos genéricos.}

Son la base de los servicios comunitarios de atención psicosocial. El objetivo es proveer un amplio rango de intervenciones psicosociales para los adultos entre 18 y 65 años con enfermedad mental crónica pertenecientes a un área determinada. El equipo trabaja para insertar a los pacientes en su medio comunitario, incrementando su funcionamiento social. Pretende asegurar la continuidad y la flexibilidad de cuidados. Los pacientes también se benefician de ver al mismo equipo durante largo tiempo, siendo un aspecto muy beneficioso cuando existe una buena relación para los momentos de crisis. La intensidad de la atención es flexible según las necesidades del paciente. Otra ventaja es que la estructura del servicio es fácil- 
mente comprensible para el resto de recursos y para el paciente. Este tipo de equipos son ideales para poblaciones entre 30.000 y 50.000 habitantes. La desventaja de proveer un amplio rango de distintas intervenciones puede ser la saturación de funciones que recaen en el mismo equipo. Esto llevaría a reducir la especificidad e intensidad de las intervenciones siendo difícil, por ejemplo, priorizar sobre los pacientes que precisan de un abordaje más específico. Otra desventaja es que cuando se termina el tiempo de trabajo se termina la atención, quedando muchos pacientes con horas libres que siguen necesitando de la atención del equipo.

\section{(2) Equipos genéricos con un equipo de PACT complementario}

El PACT consiste en un equipo multidisiciplinar (psiquiatras, enfermeros, psicólogos, terapeutas ocupacionales, etc.) que provee servicios todos los días de la semana. La atención tiene lugar en el ambiente del paciente, mediante contactos frecuentes, visitas y actuaciones «in vivo». El ratio de pacientes por profesional es pequeño y está entre 1:10 ó 1:15. El equipo se ocupa de las dificultades interpersonales de los pacientes, provee recursos materiales básicos, trabaja con los familiares o personas próximas, resuelve los problemas de la vida diaria, mantiene a los pacientes en el programa evitando abandonos, evita rehospitalizaciones y enseña habilidades instrumentales necesarias para la vida independiente. El equipo está coordinado con otros recursos facilitando el acceso y el trabajo complementario en caso de que el paciente lo precise.
En el ámbito rural la organización del programa tendría que contemplar algunas consideraciones en aras de su eficacia. Siguiendo al UK 700 Group (33) cada profesional o CM debería tener menos de 15 pacientes. Por otra parte la evidencia permanece poco clara respecto a si el PACT es la solución para todo tipo de pacientes y de localidades. Probablemente, el modelo tendría cabida en localidades inaccesibles y con pacientes que rechazan otra clase de servicios. También hay que tener en cuenta que si los servicios locales son escasos y están fragmentados, la capacidad del equipo se verá limitada, pues sus acciones se desvanecerán ante la imposibilidad de sustituir la cobertura mínima necesaria (hospitalizaciones, alojamientos, clubs de ocio, grupos de ayuda, etc.).

Lo más indicado y atendiendo a los datos, sería que el PACT atendiera solo a un subgrupo de pacientes determinados de la comunidad rural (entre 100 y 150). También es necesaria la coordinación entre los dos equipos.

EL PACT debiera considerarse más como una forma de organizar los tratamientos que como un tratamiento en sí mismo.

\section{(3) Equipos genéricos con funciones de PACT añadidas}

Este modelo consiste en un equipo comunitario multidisciplinar genérico con funciones de PACT. El equipo tendría que dedicar parte de su tiempo a las tareas de atención asertiva comunitaria. Una variante de este tipo de organización pero más encaminada al «case-management» ha sido descrita en nuestro país por Ximénez, 
Fernández y Sánchez (34) como un programa de rehabilitación y seguimiento de pacientes enfermos mentales crónicos para garantizar la continuidad de cuidados y favorecer su integración en la comunidad.

Como inconveniente de este modelo en el ámbito rural está el hecho de que compatibilizar en el mismo equipo la atención en el centro con los desplazamientos para realizar intervenciones, puede conllevar una disminución de la eficiencia , así como un gran gasto de tiempo asistencial unido a una escasa cantidad de pacientes a los que se puede llegar.

\section{(4) Equipos genéricos con un equipo de atención a domicilio}

En la literatura ha sido descrito como una combinación entre un equipo genérico y un equipo de atención a domicilio para las crisis (35-37).

En el contexto rural la presencia de un equipo de atención a domicilio tendría entre sus acciones más ventajosas, no solo la atención durante las crisis, sino, detectar a los enfermos mentales que no frecuentan los servicios, proveer de recursos materiales necesarios, trabajar con la red social del paciente y poner al paciente en contacto con recursos comunitarios (empleo, ocio, cultura, etc.). Iría orientado a los pacientes que permanecen inaccesibles.

Una ventaja importante es que el equipo no tiene que tener una formación específica en salud mental, siendo su atención en el domicilio más un «enlace» para personas con enfermedad mental en riesgo de marginación o exclusión. Por tanto la contratación de paraprofesionales que pertenezcan a la comunidad del paciente es una alternativa interesante. Este modelo encajaría bien en un sistema donde los servicios especializados están alejados de los servicios comunitarios generales.

La descoordinación entre el equipo domiciliario con los otros recursos, o la infravaloración del mismo por los servicios de salud mental es una desventaja que habría que tener en cuenta.

\section{(5) Equipos genéricos con especialistas integrados en el equipo}

Este modelo consiste en desarrollar un equipo o miniequipo especializado en intervenciones necesarias y ajustadas a la población atendida por el equipo genérico. Puede ser, como ejemplo, mediante la contratación de profesionales especialistas en la atención en crisis, en la atención a domicilio o en la inserción laboral. Esta idea surge debido a la especialización común que se requiere en la práctica diaria de la rehabilitación psicosocial (inserción laboral, apoyo familiar, entrenamiento en AVD, manejo de síntomas resistentes, etc.)

Como hemos visto hasta el momento, todos estos modelos consisten en distintas variedades de programas comunitarios. Aunque en algunos casos no existe evidencia clara de cada modelo (37) existe una acumulación de datos que hacen pensar que la implementación de cualquiera de ellos obtendrá beneficios en aspectos como: hospitalizaciones, funcionamiento social y satisfacción (32).

Aunque los centros genéricos de rehabilitación psicosocial obtienen beneficios, parece claro que en «lo rural» precisarán de alguna adaptación, siendo una referencia para ello alguna de las alterna- 
tivas aquí comentadas. Lo importante consiste en adaptar el recurso a la rutina y particularidad local. El equipo de Bond (38, 39) fueron pioneros en comenzar con estas experiencias de adaptación, pues, partiendo de la atención desde un Centro de Rehabilitación psicosocial, adaptaron sus programas a la realidad de su territorio, contemplando la coordinación, la intervención en la comunidad y la continuidad como principios básicos.

\section{Fidelidad de los modelos de atención:} Ingredientes esenciales para una atención rural comunitaria

La dificultad y complejidad del medio rural exige el desarrollo de unos criterios que faciliten la implantación de los programas con una buena relación costeeficacia. Las localidades que asuman alguno de los modelos expuestos deben autoevaluarse para comprobar si están ofreciendo intervenciones que han demostrado eficacia y pertinencia.

En realidad, no está claro todavía qué tipo de ingredientes asegurarán la eficacia de la intervención. El «UK 700 Group» (33) sugiere los siguientes: ratio profesional-pacientes reducido (1:15), existencia de recursos sociosanitarios que apoyen y complementen al programa $\mathrm{y}$, definición clara del perfil del paciente que va a ser atendido por el programa.

Issakidis (30) encontraron en su estudio con 73 enfermos mentales graves que el factor que podía predecir la eficacia del programa es el ratio profesional-paciente, ya que el profesional que tenga un ratio entre $1: 10 / 15$ puede desarrollar intervenciones cualitativamente distintas de otro que tenga 1:30 pacientes.

Teague y otros (31) enfatizaron la importancia de documentar los componentes de tratamiento que proveen eficacia para los programas asertivo-comunitarios, criterios que también han sido usados para evaluar los programas de «casemanagment» intensivos (26). Recientemente estos autores han desarrollado una escala para medir la fidelidad de los tratamientos a la hora de aplicarlos (tabla 2).

Tabla 2. Criterios de fidelidad para el PACT. (Teague, et al)

\begin{tabular}{l} 
- Proporción de casos asignados a cada profesional 1:10 \\
\hline - E equipo comparte casos \\
\hline - Existen reuniones del equipo \\
\hline - E equipo tiene un líder o responsable \\
\hline - Continuidad de la dotación y de los miembros del equipo \\
\hline - E equipo cuenta con un psiquiatra \\
\hline - E equipo cuenta con un enfermero \\
\hline - E equipo cuenta con un especialista en drogodependencias \\
\hline - E equipo cuenta con un especialista en actividades vocacionales \\
\hline - Se han definido criterios de inclusión en el programa \\
\hline - Tasa de ingresos en el programa \\
\hline - Responsabilidad completa del tratamiento \\
\hline
\end{tabular}

\begin{tabular}{l} 
- Responsabilidad de intervención en crisis \\
\hline - Responsabilidad de planificar el alta \\
\hline - Seguimiento por tiempo ilimitado \\
\hline - Intervención «in vivo» (ej. en el domicilio) \\
\hline - Política de evitar abandonos \\
\hline - Seguimiento asertivo \\
\hline - Intensidad de servicios \\
\hline - Frecuencia de contactos alta \\
\hline - Trabajar con el sistema de soporte \\
\hline - Tratamiento de la adicción por el mismo equipo \\
\hline * Tomado de Hernández(26)
\end{tabular}



INFORMES

Un enfoque más específico en al ámbito rural acerca de los ingredientes que pueden aumentar la potencia de las intervenciones nos permitiría acercarnos con mayor claridad a los ingredientes que son necesarios para esperar los mejores resultados posibles. Este es un área que ha reci- bido escasa o ninguna atención en la literatura. Santos et al.(13) compararon las características de los programas comunitarios tradicionales con los programas asertivo-comunitarios en áreas urbanas y rurales (ver tabla 3 ).

Tabla 3. Comparación de las características entre programas tradicionales y PACT en áreas rurales y urbanas.

\begin{tabular}{|c|c|c|c|}
\hline Caracteristicas del servicio & Programas comunitarios tradicionales & \multicolumn{2}{|c|}{ PACT } \\
\hline \multirow[b]{2}{*}{ Proveedor de servicio } & \multirow[b]{2}{*}{ Un clínico (individual) } & Urbano & Rural \\
\hline & & Compartida por el equipo & $\begin{array}{l}\text { Equipo con ayuda de } \\
\text { voluntario de la comunidad }\end{array}$ \\
\hline Ciente (consumidor del servicio) & Paciente (individual) & Varios casos (servicios compartidos) & Varios casos (servicios compartidos) \\
\hline Proporción de profesionales:pacientes & De 1:30 a 1:50 & De 1:10 hasta 1:12 & De 1:10 hasta 1:12 \\
\hline Comunicación en el equipo & Infrecuente & Reuniones diarias & Reunión 2 veces por semana \\
\hline Disponibilidad del equipo & Limitada a las horas de despacho & 24h. al día, 7 días de la semana & $\begin{array}{l}\text { Atención de día con disponibilidad de } \\
\text { 24h. para consultas telefónicas. }\end{array}$ \\
\hline Lugar de tratamiento & Cínica & Comunidad & Comunidad \\
\hline Lugar de rehabilitación & Contextos facilitadores & Comunidad & Comunidad \\
\hline Tratamiento de base & Psicoterapiay medicación & $\begin{array}{l}\text { Hexible e individualizado (ajustado } \\
\text { a necesidades) }\end{array}$ & $\begin{array}{l}\text { Pexible e individualizado (ajustado } \\
\text { anecesidades) }\end{array}$ \\
\hline Frecuencia de contacto & 1 vez cada mes 03 meses & Varios por semana (cada2 ó 3 días) & 1 vezala semana \\
\hline Frecuencia de contacto con la familia & Ocasionalmente & 1/semana & $\begin{array}{l}\text { 1/semana coincidiendo con lavisita } \\
\text { a domicilio }\end{array}$ \\
\hline Adherenciaa la medicación & Ocasionalmente & Lo indicay administra el equipo & $\begin{array}{l}\text { Responsabilidad compartida entre el } \\
\text { paciente, el equipo y lafamilia }\end{array}$ \\
\hline Quidado de lavivienda & $\begin{array}{l}\text { Responsabilidad del paciente } \\
\text { y lafamilia }\end{array}$ & Responsabilidad del equipo & $\begin{array}{l}\text { Responsabilidad compartida entre } \\
\text { el paciente, el equipo y lafamilia }\end{array}$ \\
\hline Funciones de los tutores ("case managers") & Organización/corretaje de casos & Provisión directa de servicios & Ambas cosas \\
\hline Pisos protegidos & $\begin{array}{l}\text { Transición graduada a vivienda } \\
\text { independiente }\end{array}$ & Máximaindependencia & $\begin{array}{l}\text { La mayoría de los pacientes vive } \\
\text { con la familia Trabajo para } \\
\text { incrementar la satisfacción con la } \\
\text { vivienday mejorar el ambientey el } \\
\text { funcionamiento del paciente. }\end{array}$ \\
\hline Movilización de recursos comunitarios & Baja & Considerable & Alta \\
\hline
\end{tabular}

-Tomada de Santos et al.(13). 
Considerando lo anterior, proporcionar una buena atención comunitaria a los pacientes rurales requiere una buena organización de los servicios. A pesar de las deficiencias estrucutrales de fondo del contexto rural es posible contar con unos criterios que favorezcan la calidad de los programas de atención psicosocial en este ámbito. Haciendo un esfuerzo integrador de los datos con los que contamos y si- guiendo alguna de las directrices marcadas por los PACT (programas que como hemos visto se adaptan en su metodología a lo rural) y por las experiencias en contextos rurales $(32,13,31,26)$ podemos establecer algunos de los ingredientes o criterios de calidad en el contexto rural para los enfermos mentales más graves. Se enumeran en la tabla 4.

Tabla 4. Criterios de calidad para el tratamiento y rehabilitación de los enfermos mentales graves en contextos rurales.

\begin{tabular}{|c|c|}
\hline CRITERIOS & AMBITORURAL \\
\hline Proporción de profesionales:pacientes & De 1:10 hasta 1:15 \\
\hline Responsabilidad del paciente & Compartida por el equipo \\
\hline Tipo de intervención & Énfasis en el entrenamiento en habilidades \\
\hline Medicación & Supervisión por el equipo \\
\hline Estructura del equipo & Combinación de modelo individualizado (tutoría de casos) e integración de roles clínicos \\
\hline Provisión de servicios & Directa. Atención directa más que "case-managment» \\
\hline Frecuencia de contacto & Varios a la semana \\
\hline Frecuencia de contacto con la familia & 1 ala semana \\
\hline Reuniones de equipo & Diarias para revisar los casos y organizar el trabajo diario \\
\hline Dirección del equipo & 1 coordinador supervisa el programa y la atención \\
\hline Continuidad de cuidados & Lo garantiza el equipo (clínicos, sociales y ocupacionales) \\
\hline Continuidad del equipo & Variable, con turnos relativamente estables \\
\hline Roles profesionales & $\begin{array}{l}\text { Equipo multidisciplinar con especialización de roles (terapia ocupacional, trabajador social, } \\
\text { psicólogo clínico, educador social y técnico de inserción laboral) }\end{array}$ \\
\hline Integración en la red & Acceso a otros recursos en la comunidad (hospital, sistemas de salud, legales, sociales, otros) \\
\hline Cobertura & Atención de día \\
\hline Perfil del paciente & $\begin{array}{l}\text { Citerios explícitos de admisión: pacientes con trastorno mental severo diagnosticados por servicios } \\
\text { de salud mental locales y con hospitalizaciones psiquiátricas previas }\end{array}$ \\
\hline Lugar de contacto & Cínica/comunidad \\
\hline Integración de tratamiento & Rehabilitación integrada con tratamiento en crisis desde una USM (con atención a domicilio) \\
\hline $\begin{array}{l}\text { Responsabilidad para admisión en } \\
\text { el hospital }\end{array}$ & Responsabilidad compartida entre clínicos y rehabilitadores \\
\hline Duración del servicio & llimitada \\
\hline $\begin{array}{l}\text { Lugar de la rehabilitación psicosocial } \\
\text { de habilidades }\end{array}$ & En el medio donde vive el paciente \\
\hline Seguimiento & Intensivo. Prevención de abandonos \\
\hline Contacto con recursos comunitarios & Alto \\
\hline Pisos protegidos & Responsabilidad del equipo \\
\hline Evaluación de resultados & Hospitalizaciones, abandonos, calidad de vida, funcionamiento psicosocial y satisfacción \\
\hline
\end{tabular}




\section{Conclusiones}

La implementación de programas de atención psicosocial para enfermos mentales graves que se han descrito en el presente artículo han mostrado una serie de ventajas importantes: reducción de hospitalizaciones, mayor satisfacción, mejora del funcionamiento social, reducción de abandonos, etc. Sin embargo estos resultados quizá no sean comparables en las áreas rurales ya que los principios activos de las intervenciones pueden operar de forma diferente debido a las condiciones particulares del medio: comunicación entre profesionales, accesibilidad, escasez de recursos, actitudes hacia la enfermedad mental, etc.

Los programas de rehabilitación psicosocial se centran típicamente en las necesidades del paciente mediante la atención directa en un centro de rehabilitación (CRPS), sin embargo, los equipos de las áreas rurales se encuentran con la dificultad de proveer los servicios a los pacientes que se encuentran a largas distancias del centro. Por tanto, el equipo rural debe planificar cuidadosamente la atención diaria que va a desarrollar dentro y fuera del centro, el tipo de pacientes al que va a atender y los desplazamientos que tendrá que hacer en ese día. Esto puede suponer una saturación que hace plantearse la necesidad de adaptar sistemas de atención en este ámbito. Un aumento desmedido de la carga asistencial hace peligrar los principios básicos de la atención integral al enfermo mental: individualización y continuidad de cuidados.

Puede decirse que en el medio rural se aprecia una insuficiencia de recursos de rehabilitación adaptados a la realidad local. Falta una definición acerca de qué servicios serían los más adecuados en cada situación concreta. Existen riesgos que pueden hacer tambalear los escasos programas de atención psicosocial, que actúan como «apagafuegos» ante las demandas producidas, no solo por la enfermedad mental, sino también por el contexto en el que se encuentran: patologías severas, escasa articulación de demandas, consulta farmacológica como eje principal de la intervención, dispersión geográfica, tolerancia familiar, ausencia de alternativas residenciales, poca coordinación, población no identificada que permanece inaccesible, etc.

Es muy difícil identificar los «ingredientes activos» y saber cuáles serán los factores críticos que aportarán los mejores resultados en cada programa. Sin embargo, sí podemos analizar cómo adaptar los servicios a cada realidad rural. Las adaptaciones deben hacerse de manera singular en cada ámbito. No obstante, los «criterios de fidelidad» definidos en este artículo pueden ser una guía para conseguir este reto. La necesidad de aplicar estos principios de forma más o menos intensiva puede ser un aspecto fundamental para el éxito o el fracaso. Se ha comprobado que la implementación parcial de criterios básicos como reuniones de equipo, accesibilidad o coordinación con otros servicios pueden ser responsables del fracaso del programa (17).

Los principios que sirven de guía para desarrollar los modelos asertivo-comunitarios y los modelos de «case-managment intensivos» se muestran adecuados para su 
consideración en el medio rural como modelos alternativos a los tradicionales, ya que entre sus principios se encuentran el apoyo comunitario, la normalización, la inserción y el trabajo en el medio. Un aspecto fundamental de cara al ámbito rural de estos modelos consiste en el esfuerzo que se lleva a cabo para mantener a los pacientes dentro del programa, evitando abandonos.
En general puede decirse que el ámbito rural es un área que requiere de un enfoque específico y singular para asegurar una adecuada atención comunitaria y ofrecer el apoyo necesario a las personas que tienen una enfermedad mental grave y crónica.

\section{BIBLIOGRAFÍA}

1. Stroul, B.A., «Community support systems for persons with long-term mental illnes: a conceptual framework», Psychosocial Rehabilitation Journal, 1989, 12(3), 9-26.

2. Rebolledo S. (coord). Rehabilitación Psiquiátrica (curso de postgrado de rehabilitación psicosocial de personas con trastornos mentales crónicos), Universidad de Santiago de Compostela, 1997.

3. Desviat,M.; Pastor, A.; Blanco, A.; Ramírez, F., Rehabilitar en la comunidad, 2001, (2),

4. Turner, J.C., «Comprehensive community support systems for mentally disabled adults: definitions components, guiding principles», Psychosicial Rehabilitation Journal, 1977, 1(3), 9-26.

5. Anthony, W.A.; Blanch, A. «Research on community suport service. What have we learned. Psychosocial Rehabilitation Journal, 990, 12(3), 55-81.

6. Lehman, A.F. ; Thompson, J.W. ; Dixon,L.B., y otros, «Schizophrenia : treatment outcomes research editors' introduction», Schizophrenia Bulletin, 1995, 21, 561-566.

7. Rodríguez, A.; Sobrino, T., «Evolución de la atención al enfermo mental crónico. La reforma psiquiátrica y el desarrollo de la atención comunitaria», En A.Rodríguez (coord.): Rehabilitación Psicosocial de personas con trastornos mentales crónicos, 1997. Madrid. Pirámide.

8. Thornicroft, G.; Susser, E., «Evidence-based psychoterapeutic interventions in the community care of schizophrenia», British Journal of Psychiatry, 2001, 178: 2-4.

9. Liberman, R.P., Rehabilitación Integral del enfermo mental crónico, 1993. Barcelona. Martínez Roca.

10. Rodríguez, A. (coord.) Rehabilitación Psicosocial de personas con trastornos mentales crónicos, 1997. Madrid. Pirámide.

11. Anthony, W.A.; Liberman, R.P., «The practice of psychiatric rehabilitation: historical, conceptual and research base», 1986, Schizophrenia Bulletin, 12, 542-559. 
INFORMES

12. Husted, J.; Wentler, S.A; Bursell, A., «The effectiveness of community support programs for resistenly mentally ill in rural areas», Community Mental Health Journal, 1994, 30 (6), 595-600.

13. Santos, A.B.; Deci, P.A.; Lachance, M.A.; y otros., «Provinding assertive community treatment for severely mentally ill patients in a rural area», Hospital and Community Psychiatry, 1993, 44(1), 34-39.

14. Tobin, M.J. «Rural psychiatric services», Aust N Z Psychiatry, 1996, 30(1), 114-123.

15. AMSM., Prestaciones básicas y estándares de calidad en los servicios de salud mental. Cuaderno Técnico, 1, 1998. Madrid. Desk Impresiones.

16. AEN. «Rehabilitación Psicosocial del trastorno mental severo. Situación actual y recomendaciones», Cuadernos técnicos, 2002, 6,. Madrid.

17. McDonel, E.C,; Bond, G.R.; Salyers, M.; y otros., «Implementing assertive community treatment programs in rural areas», Administration and Policy in Mental Health, 1997, 25(2), 153-173.

18. Sullivan, G.; Jackson, C.A.; Spritzer, B.A., «Characteristics and service use of seriously mentally ill persons living in rural areas», Psychiatric Services, 1996, 47(1), 57-61.

19. Simón, M., «Accesibilidad y Rehabilitación: la accesibilidad en contextos rurales», Jornadas sobre salud mental: problemas de adaptación e integración social de la persona con enfermedad mental crónica, 2002, Madrid. Documento técnico publicado por el IMSERSO-Ministerios de Trabajo y Asuntos Sociales.

20. Yuen, E.J.; Gerdes, J.L.; Gonzales, J.J.; «Patterns of rural mental health care.An exploratory study», Gen Hosp Psychiatry, 1996, 18(1), 1-2.

21. Hartley, D., «Effects of managed mental health care on service use in urban and rural Maine», Journal of Rural Health, 2001, 17(2), 95-104.

22. Flaskerud, J.H.; Kvitz, F.J., «Resources rural consumers indicate they would use for mental health problems», Community of Mental Health Journal, 1982, 18(2), 107-109.

23. Cohen, J., «The effect of distance on use of outpatient services in a rural mental health center», Hospital and community Psichiatry, 1972, 23, 27-28.

24. Meise, U.; Kemmler, G. ; Kurz, M.; y otros, «Quality of the location as a principle in psychiatric health care planning», Gesundheithswesen, 1996, 58(1), 29-37.

25. Thornicroft, G.; Wykes, T.; Holloway, F.; y otros, «From efficacy to effectiveness in comunity mental health services», British Journal of Psychiatry, 1998, 173, 423-427.

26. Hernández-Monsalve, M., «Los programas de seguimiento intensivo en la comunidad: una alternativa a la hospitalización ¿y algo más?», en F.Rivas Guerrero (coord.): La Psicosis en la comunidad, 2000, Madrid. AEN.

27. Workman, E.A.; Shorth, D.; Turner, R.; y otros, «A 30-year progress report on a VA Satellite psychiatric clinic program», Psychiatric Services, 1997, 48(12), 1582-1583.

28. Penn, D.L.; Mueser, K.T.; «Research update on the psychosocial tretament of schizophrenia», American Journal of Psychiatry, 1996, 153, 607-617. 
INFORMES

29. Burns, B.J.; Santos, A.B., «Assertive community treatment:: an update of randomized trials», Psychiatric Services, 1995, 46, 669-675.

30. Issakidis, C.; Sanderson,K.; Teeson, M.; y otros, «Intensive case managment in Australia: a radomized controlled trial», Acta Psychiatrica Scandinavica, 1999, 99, 360367.

31. Teague, G.; Bond, G.; Drake, R., «Programme fidelity in assertive community treatment: development and use of a measure», American Journal Orthopsychiatry, 1998, 68, 216-232.

32. Thornicroft, G.; Becker, F.; Holloway, S.; y otros, «Community mental health teams: evidence or belief?», British Journal of Psychiatry, 1999, 175, 508-513.

33. UK 700 Group, «Comparison of intensive and standard case managment for patients with psychosis», British Journal of Psychiatry, 1999, 174, 74-78.

34. Ximénez, P.; Fernández, C. ; Sánchez, A., «Un programa de seguimiento y cuidados comunitarios de personas que padecen enfermedad mental crónica», Trabajo Social hoy, 2000, 28, 77-87.

35. Hoult, J., «Community health of the acutely mentally ill», British Journal of Psychiatry, $149,137-144$

36. Test, M.A.; «Training in comunity living», en R.P. Liberman (coord): Handbook of Psychiatry Rehabilitation, 1992, 153-170. New York. Mcmillan.

37. Mueser, K.T.; Bond, G.R.; Drake, R.E.; y otros, «Models of community care for severe mental illness: a review of research on case managment», Schizophrenia Bulletin, 1998, 24, 37-74.

38. Bond, G.R,; Miller, L..D.; Krunweid, R.D.; y otros, «Assertive case managment in three CMHCs. A controlled study», Hosp Comm. Psychiatry, 1988, 39, 411-418.

39. Bond, G.R.; Witheridge, T.G.; Wasmer, D.; y otros, «A comparison of two crisis housing alternatives to psychiatric hospitalization», Hosp Comm. Psychiatry, 1989, 40, 177183.

* Psicólogo clínico. Coordinador del programa de Centros de Rehabilitación Psicosocial y Laboral de FISLEM. Castilla La Mancha. 